\title{
EUROPA: ESTRUCTURA INSTITUCIONAL PARA LA SEGURIDAD DESDE LA PAZ DE WESTFALIA
}

EUROPE: INSTITUTIONAL STRUCTURE FOR SECURITY SINCE THE PEACE OF WESTPHALIA

\author{
Francisco José Blanco Jiménez \\ Alberto Romero Ania \\ Universidad Rey Juan Carlos, Madrid. España/Spain \\ francisco.blanco@urjc.es \\ alberto.romero@urjc.es
}

Recibido/Received: 05/05/08

Aceptado/Accepted: 14/07/08

\section{RESUMEN}

El presente trabajo pretende abordar una revisión sucinta de los acontecimientos más significativos del proceso histórico que ha determinado la estructura institucional para la seguridad en Europa desde la Paz de Westfalia. Europa es la región del mundo con mayor nivel de coordinación y cooperación multilateral. La idiosincrasia y el espíritu de cooperación de los pueblos europeos son fruto de la evolución histórica común y del convencimiento general de que un marco institucional estable garante de la seguridad, junto con la cooperación económica y política, son el mejor baluarte del Estado de bienestar y de la prosperidad de Europa.

\section{PALABRAS CLAVE}

Economía institucional, historia, geopolítica, Europa.

\begin{abstract}
The present work seeks to carry out a succinct revision of the most significant events in the long historical process that have determined the institutional structure for the security in Europe from the Peace of Westphalia. Europe is the region of the world with the highest level of coordination and multilateral cooperation. The idiosyncrasy and the spirit of cooperation of the European States is based on the long common history process and on the general understanding that an efficient institutional framework is the best guarantee for security, as well as the economic and political cooperation among the States, which enhance the European peace and prosperity.
\end{abstract}

\section{KEYWORDS}

Institutional economy, history, geopolitics, Europe. 


\section{INTRODUCCIÓN}

La situación de bienestar, paz, prosperidad, cohesión, cooperación y armonía entre los pueblos europeos son el patrimonio y la herencia más importante de la historia geopolítica e institucional de Europa. Europa es la región del mundo con mayor estabilidad y prosperidad, al mismo tiempo que es el área geográfica que cuenta con el entramado institucional más multilateral, complejo y eficiente.

La evolución histórica hacia el actual marco institucional europeo en el que la seguridad y la cooperación económica y política entre los Estados está totalmente garantizada y responde a la evolución de la estructura geopolítica, social, económica e institucional desde la creación del concepto de Estado-Nación en el siglo XVII hasta nuestros días. La seguridad, como garante y condición indispensable para el desarrollo cultural y para el crecimiento económico de los Estados, ha sido el propulsor desde el fin de la II Guerra Mundial de la creación de las instituciones a las que actualmente pertenecen los Estados europeos.

Desde el ámbito de las ciencias sociales merece especial atención el estudio de las instituciones, debido a que éstas son los mecanismos de orden social y cooperación que gobiernan el comportamiento de la sociedad mediante la elaboración e implantación de reglas.

En el estudio expuesto a continuación se analizan las instituciones políticas con funciones sociales y económicas europeas que han velado por el mantenimiento de la seguridad en el continente, al igual que su estructura, mecanismos formales de gobierno y las causas de su creación, además de la repercusión que cada una de ellas ha tenido en la evolución hacia la actual estructura institucional europea, que garantiza el crecimiento económico y el desarrollo cultural en Europa.

\section{LA PAZ DE WESTFALIA}

La paz de Westfalia supuso el fin de la Guerra de los Treinta Años en Europa, que duró desde 1618 hasta 1648. El tratado de paz influyó enormemente en la ulterior historia de Europa, debido a que la noción de un imperio católico romano ${ }^{1}$ espiritualmente dirigido por el Papa y gobernado por el Emperador, quedó definitivamente abandonada.

Como consecuencia comienza el desarrollo del concepto moderno de Europa como comunidad de Estados independientes. Significa tanto el nacimiento del concepto de "Estado-Nación" como el nacimiento de la legitimidad de las Naciones para gobernar su territorio con soberanía plena, sin interferencias externas en materias de política o religión, en contraste con la época anterior. Nace un nuevo orden internacional en el que los propios Estados deben resolver ellos mismos sus problemas de soberanía, y ser los reguladores de los conflictos internacionales mediante instrumentos de moderación y coordinación (Janis, 2004:162-170). 
Algunos de los elementos claves de los acuerdos desarrollados desde finales del siglo XVII han dado lugar al concepto moderno del sistema de coordinación internacional entre los Estados. Antes de la Paz de Westfalia los Estados no tenían ni bien definidas sus fronteras ni el monopolio sobre el uso de la fuerza, por lo que la situación de guerra ininterrumpida era la norma habitual en términos de relaciones internacionales. Por el contrario, después de la Paz de Westfalia los Estados se componen de un territorio definido, una población estable y una soberanía que les otorga una autoridad exclusiva que no permite ninguna interferencia externa en la esfera de su jurisdicción territorial. Esta soberanía les permite el uso de la fuerza en la defensa de sus intereses (Jazbec, 2006:1-4).

\section{EL CONCEPTO DE EQUILIBRIO DE PODERES}

El modelo básico de relaciones internacionales en el periodo posterior a la Paz de Westfalia fue el concepto de equilibrio político y de poderes entre los Estados europeos. La hegemonía del poder cambiaba constantemente entre los Estados más poderosos, tales como Portugal, España, Holanda, Inglaterra y Francia. Dentro de este sistema los Estados buscaban mantener un equilibrio de poderes que evitase la hegemonía de unos sobre otros.

Un elemento crucial de este sistema era la flexibilidad de los Estados para cambiar sus acuerdos y alianzas de política exterior según mandaban las circunstancias, con el objetivo de mantener el equilibrio de poderes. Este equilibrio de poderes aparece en el transcurso del siglo XVIII, un periodo propicio para ello, dada la situación geopolítica dominante en Europa marcada por la ausencia de nacionalismos como determinantes principales de las decisiones en materia de política exterior. Esta época se caracteriza por el desarrollo de los Estados-Nación soberanos, y con voluntad política de limitar los enfrentamientos militares. Igualmente, se produce un enorme retroceso del fanatismo e intolerancia religiosa omnipresente a lo largo todo el siglo precedente.

Durante el siglo XVIII se produjeron numerosas contiendas bélicas en Europa por la necesidad de mantener el equilibrio de poderes entre los Estados (Buzán y Waever, 2003:97-102). Un ejemplo de estas luchas por mantener el equilibrio político militar en Europa fue la guerra de Sucesión Española, que abrió el siglo XVIII. Por un lado fue un conflicto internacional por la sucesión al trono de España e intentar frenar la expansión de Francia, y por otro lado, las potencias europeas querían evitar el resurgimiento del eje España-Austria que existió en tiempos de Carlos I. En aquella época Inglaterra era el único país de Europa cuya razón de Estado no era la expansión en el continente, sino el mantenimiento del equilibrio de poderes. Con este mismo propósito, y especialmente para frenar la expansión francesa, se creó la Gran Alianza, También denominada Alianza de la Haya, en 1702 entre Austria, Inglaterra, Holanda y Dinamarca, a la que se unieron en 1703 Portugal y Saboya. A pesar de la guerra entre la coalición y Francia, 
Francia se consolidó como la gran potencia de Europa, aunque no poseía capacidad de dominio sobre otros Estados ${ }^{2}$ (Kissinger, 1994:17, 70-71).

\section{LA PAZ DE UTRECHT}

En los Tratados de Utrecht firmados en 1712 y de Rastadt en 1714, Europa cambió su mapa geopolítico estableciendo la paz en el continente. Estos tratados se consideran, también, el fin de la Guerra de Sucesión Española (1702-1714). Durante la primera mitad del siglo XVIII se produjo una situación de poder bipolar manteniendo una tensión entre los Estados europeos. Con el transcurrir del siglo XVIII la atención de las relaciones internacionales se centró en los intereses comerciales y coloniales, especialmente en lo que se refiere a Gran Bretaña (Buzan y Weaver, 2003:115).

\section{EL SIGLO XIX}

El siglo XIX muestra un gran contraste, tanto con el periodo revolucionario anterior, como con la era del bienestar alcanzada en Europa en el siglo posterior. Este periodo se distinguió por la estabilidad general y la ausencia de guerras sistemáticas y generalizadas. La política exterior de las grandes potencias estuvo caracterizada por la cautela, aunque esto no evitó que la amenaza del uso de la fuerza fuese un recurso frecuente durante este periodo. Los Estados europeos optaron generalmente por la diplomacia como instrumento preferente para resolver los conflictos, seguido del uso de sanciones, y en último lugar la intervención militar, entendida ésta como un instrumento legítimo de la política exterior (Buzan y Waever, 2003:122).

El siglo XIX se puede dividir en tres periodos de tiempo bien diferenciados. El primer periodo está determinado por el restablecimiento de las fronteras de Europa, impuestas tras el Congreso de Viena de 1815, que se firma tras el fin de las guerras napoleónicas en Waterloo. Este periodo caracterizó la diplomacia europea durante las siguientes cuatro décadas. El segundo periodo estuvo dominado por las políticas del Canciller de Hierro alemán (Otto von Bismarck, 1815-1898) aplicadas durante los años setenta y ochenta, y el tercer periodo comenzó después de la destitución del Canciller Bismarck, hasta el inicio de la I Guerra Mundial.

El sistema de relaciones internacionales consensuado por los líderes europeos y aplicado en el Viejo Continente tras el Congreso de Viena, establecía un nuevo orden internacional en el que quedaba restaurado el equilibrio de poderes y se regulaba la resolución de disputas por medio de conferencias internacionales. Los dirigentes europeos construyeron un nuevo orden postbélico con el objetivo de corregir las debilidades del sistema precedente. Los Jefes de Estado europeos habían aprendido durante los conflictos acaecidos en el último cuarto de siglo, que Europa necesitaba de los mecanismos de creación de una coalición u organización 
de respuesta automática ante agresiones. Estos nuevos conceptos y prácticas en política exterior europea serían la semilla ideológica de La Sociedad de Naciones (League of Nations), y posteriormente de las Naciones Unidas.

El sistema de alianzas, basado nuevamente en el concepto de equilibrio de poderes, incluía el concepto de "legitimidad compartida para acciones comunes". Un ejemplo relevante de esta idea es la Santa Alianza, acordada en 1815 entre Rusia, Austria y Prusia. Esta alianza se convirtió en el llamado Sistema Metternich, que otorgaba la posibilidad de poder intervenir militarmente en cualquier país contra movimientos revolucionarios. Sin embargo, el exponente del concepto "seguridad común" más importante de la época, lo constituyó el pacto de la Cuádruple Alianza, suscrita por Austria, Prusia, Rusia e Inglaterra. Esta alianza se convertiría en el verdadero árbitro de la situación internacional, origen de la llamada "práctica de los Congresos", que preveía la celebración periódica de conferencias cuyo objetivo era mantener la paz y custodiar los intereses comunes en Europa. Durante los congresos de la Cuádruple Alianza que se celebraron entre 1818 y 1822, se decidían las medidas a adoptar frente a la aparición de movimientos y revueltas de carácter liberal y nacionalista. Destacan los Congresos de Aquisgrán, Troppau, Laibach y el de Verona, en el que se acordó en 1822 la intervención en España de un ejército apodado los "Cien Mil Hijos de San Luis", para restaurar en el trono absolutista al rey Fernando VII. En el año 1818 Francia recuperó su protagonismo en el sistema internacional al ser incluida como Estado soberano miembro de la llamada, a partir de entonces, Quíntuple Alianza.

Este sistema de congresos supuso un gran avance en la cooperación europea y en las acciones de seguridad conjuntas. Se diferencian del futuro Consejo de Seguridad de las Naciones Unidas en que estaban constituidos únicamente por las potencias más poderosas. El largo periodo de paz propiciado por el Congreso de Viena estaba basado en tres pilares: la paz y la conciliación con Francia, que le otorgaba la posibilidad de actuar como un Estado soberano en igualdad de condiciones; un equilibrio político y de poderes gracias al papel determinante de la Santa Alianza y la Cuádruple Alianza; y un sentido compartido de legitimidad basado en valores comunes que refutaban las amenazas de alterar el orden internacional impuesto (Kissinger, 1994:242).

En consecuencia Europa disfrutó del periodo más largo de paz que nunca antes había conocido, en el que hubo ausencia de guerras entre las grandes potencias durante cuarenta años. El triunfo de los nacionalismos en Alemania e Italia a principios de los años setenta eliminó del mapa la mayoría de los pequeños Estados que todavía existían en Europa. Esta nueva era estuvo caracterizada por una gran rivalidad comercial e industrial, el aumento de los nacionalismos, y el crecimiento de la influencia militar en la formulación de los objetivos de la política exterior (Buzan y Waever 2003:133-134).

Se produce un cambio ideológico en las relaciones internacionales y en la política exterior, pasando del término raison d'état al término Realpolitik, que basa las relaciones internacionales en cálculos de poder y en el interés nacional 
aplicados ad casum (Kissinger, 1995:130). Con la creación del Imperio Alemán a finales del siglo XIX, Alemania se convierte, al unirse más de 30 Estados germanos, en el más poderoso de los Estados europeos desde un punto de vista económico y militar. Este hecho revolucionó la diplomacia europea, debido a que hasta aquel momento las potencias periféricas, habían presionado y dominado siempre el centro de Europa. Fruto de tales cambios surgió tanto la rivalidad entre Francia y Alemania por Alsacia y Lorena, territorios muy ricos en minería, como la rivalidad entre el Imperio Austro-Húngaro contra Rusia por el dominio del sureste de Europa.

Igualmente aparece un nuevo tipo de consenso internacional en Europa, el de la "alianza por resignación". Esta fue la política exterior llevada a cabo por el Canciller Bismarck, que haciendo uso de la buena reputación del poderío militar alemán no otorgaba otra opción a los Estados que la de aceptar la oferta de alianza (Farmer, 2000:43-48). Con este tipo de negociaciones en 1879 Alemania consiguió crear una Doble Alianza con Austria-Hungría para ofrecerse ayuda mutua ante un eventual ataque de Rusia, y posteriormente en 1882 se amplió a una Triple Alianza con Italia. Sin embargo, a pesar de los acuerdos firmados, el Canciller Bismarck se reservaba el derecho a la intervención y actuaciones diplomáticas individuales (Buzan y Waever, 2003:134).

El equilibrio de poderes entre las dos grandes coaliciones caracterizó una etapa de gran inestabilidad conocida como la Paz Armada entre 1882 y 1914. Como resultado de estas tensiones, se crearon vastos sistemas de alianzas. De una parte Francia, el Imperio Británico y el Imperio Ruso, que formarían la Triple Entente, y por otro lado el Imperio Alemán, el Imperio Austrohúngaro e Italia formando la Triple Alianza. Estas dos coaliciones altamente hostiles e inflexibles en sus posturas se vieron enfrentadas en un tenso periodo de maniobras políticas y diplomáticas, que intentaban ganar aliados para estar preparados ante un inevitable enfrentamiento futuro. Durante esta época las naciones europeas transformaron el concepto de equilibrio de poder en el de "carrera armamentística", sin entender que los avances tecnológicos de la época implicaban la mayor amenaza jamás soportada por las naciones europeas (Kissinger, 1994:169).

Aunque la época entre 1814 y 1914 se caracterizó por dos de los tres periodos de paz más largos en la historia de Europa, el primero de 1815 a 1853 y el segundo de 1871 a 1914, la eliminación y censura de la guerra como solución de los conflictos no fue un objetivo principal para los gobiernos del siglo XIX, que veían la guerra y la amenaza militar como aceptables y legítimas, si no amenazaban la estabilidad del sistema (Buzan y Waever, 2003:35-136). Este pensamiento contrasta con el nuevo orden internacional impuesto tras la Paz de Versalles.

Antes de la I Guerra Mundial Gran Bretaña era la potencia marítima más importante y Londres el principal centro financiero del mundo. Sin embargo esto no era suficiente para imponer su hegemonía en el continente. Ante la necesidad de aliados en Europa para frenar la expansión alemana y rusa, Gran Bretaña creó en 1904 la Entente Cordiale con Francia. Este acuerdo permitía a Gran Bretaña, que 
ya disponía de inmunidad ante invasiones extranjeras por su superioridad naval, asegurarse el equilibrio de poderes en el continente para evitar el nacimiento de una superpotencia que pudiera representar una amenaza en el futuro (Brzezinski, 1997:20-21).

\section{LA PAZ DE VERSALLES}

El concepto de seguridad colectiva impuesto tras la Paz de Versalles supone un nuevo orden internacional. Tras el fin de la I Guerra Mundial con la conferencia de Paz de París en 1919, los Estados Unidos y las grandes potencias europeas decidieron crear un nuevo orden internacional, basado en un nuevo y revolucionario principio bajo la propuesta de Thomas Woodrow Wilson (18561924), presidente de los Estados Unidos. Se produjo un cambio del clásico sistema de equilibrio entre poderes por un nuevo sistema denominado a partir de entonces "comunidad de naciones". Este nuevo sistema estaba basado en la responsabilidad común por la seguridad colectiva y la oposición conjunta ante la osadía de una nación de violentar la paz conseguida.

El nuevo sistema incluía la resolución pacífica de las controversias y disputas internacionales bajo el amparo de la ley internacional (Galpin, 1995:5). Según este nuevo sistema de comunidad de naciones impuesto en Europa el nuevo orden de seguridad global ya no podría ser alterado unilateralmente por ningún Estado, y la paz debía ser alcanzada no únicamente para asegurar la defensa de los intereses del propio Estado, sino como objetivo y concepto legal en sí mismo. La custodia del sistema establecido y la responsabilidad de determinar cuándo se infringía el nuevo concepto legal debería ser acometida por una institución internacional, que fue definida como La Sociedad de Naciones, en adelante SDN (Kissinger, 1994:222).

En 1919 se reunieron en la Conferencia de Paz de París, para firmar el Tratado de Versalles, líderes políticos de 32 países. Las principales decisiones fueron tomadas por el Consejo de los Cuatro, que estaba compuesto por los Jefes de Estado de Gran Bretaña, Francia, Italia y Estados Unidos (Farmer, 2000:95). El acuerdo alcanzado en Versalles tenía muchas similitudes con los acuerdos de paz alcanzados en Utrecht en 1713 y en Viena en 1815, aunque éstos fueron mucho más eficientes. La similitud principal se refleja en el deseo de los firmantes de restablecer un nuevo orden en Europa, ofrecer una mayor estabilidad, y construir un nuevo marco internacional en el que sea más difícil para un Estado romper el consenso creado (Buzan y Waever, 2003:123).

La gran debilidad del Tratado de Paz de Versalles fue la incapacidad de restablecer un sistema económico europeo que permitiese la recuperación económica de los países derrotados. El diseño de una política macroeconómica bien estructurada y de relaciones comerciales beneficiosas entre los diferentes Estados europeos, incluyendo la plena participación de Alemania, hubiese sido necesario para asegurar la paz y la estabilidad en las siguientes décadas. 
Sin embargo, los vencedores decidieron no solamente declarar que Alemania era el único responsable del comienzo de la I Guerra Mundial, sino que acordaron aislarla y castigarla económicamente, en vez de reintegrarla en el sistema económico europeo. Alemania, que al término de la guerra tenía sus infraestructuras físicas, sociales, políticas y económicas enormemente debilitadas, fue castigada de nuevo con sanciones económicas, desarme militar, y pérdida de territorio, para reparar los daños causados a los países vencedores. Esta marginación económica llevó a la nación alemana a la gran depresión de los años treinta, lo que finalmente supuso un hecho crucial para el estallido de la II Guerra Mundial (Kissinger: 1994:239). Alemania no tuvo más opción que aceptar las cláusulas impuestas ante la amenaza de una invasión (Farmer, 2000:99).

Existen dos diferencias principales entre el orden internacional impuesto tras el Tratado de Viena y tras el Tratado de Versalles. La primera de ellas es la fuerza residual de los vencidos al finalizar la guerra, y la segunda consiste en la unión de los vencedores en 1815 mediante la Cuádruple Alianza, mientras que en 1919 los vencedores no continuaron aliados (Kissinger, 1994:228-229).

Para dar paso al nuevo sistema de seguridad y responsabilidad colectiva propuesto por el Presidente Wilson, debían darse ciertos prerrequisitos. La seguridad colectiva global necesita de los esfuerzos de la comunidad internacional para restablecer el orden y la seguridad en los enfrentamientos, tanto internos como externos, en los que el Estado falla como garante de la paz, y cuyo resultado no puede ser tolerado por la comunidad internacional. Según la Carta de las Naciones Unidas, en adelante NNUU, la única institución que puede proporcionar la seguridad colectiva son las NNUU, y en particular su Consejo de Seguridad, en adelante CSNU. El CSNU puede delegar sus funciones en organizaciones regionales por autorización expresa (Maull, 2005:14,22).

El sistema de seguridad colectivo determina sanciones conjuntas contra el país infractor perteneciente al sistema. Este sistema está basado en el acuerdo previo de sus miembros en sancionar a cualquiera de los mismos que viole ciertas normas y principios consensuados con anterioridad. Por lo que un requisito para el buen funcionamiento de un sistema de seguridad colectivo es su carácter universal y la pertenencia al mismo del mayor número de Estados posible. Otro prerrequisito debe ser un sistema de gestión en el que exista un equilibrio político y de poderes, de manera que ningún Estado pueda imponerse sobre el resto (Neuhold, 2000:7477). Respecto a los prerrequisitos legales más significativos, destaca que el funcionamiento del sistema de seguridad común implica la prohibición del uso de la fuerza con motivos no defensivos por ninguno de los Estados miembros, y la obligación conjunta de tomar medidas al respecto si se diese el caso. A nivel organizativo es preferible la centralización del sistema, contando con un órgano decisorio de pocos miembros en el que estén incluidas las principales potencias.

Este órgano puede decidir sobre si se ha incumplido alguna de las reglas de la organización por algún Estado miembro, y en tal caso sobre qué tipo de sanción debe ser impuesta. El requisito crucial de su funcionamiento debe ser la solidaridad 
internacional, en la que cada Estado miembro debe velar por la paz mundial y la seguridad global del sistema como un objetivo en sí mismo, y no únicamente cuando se vean afectados sus intereses directos. Los Estados, convencidos de que salvaguardar la paz del sistema en su conjunto implica defender indirectamente sus intereses, deben actuar con imparcialidad y acatar tomar parte en las sanciones impuestas contra otros Estados miembros, sin valorar su relación geoestratégica con el país infractor. A diferencia de lo que sucede con las NNUU, la SDN no cumplía los requisitos necesarios para el buen funcionamiento de un sistema de seguridad colectivo.

\section{LA SOCIEDAD DE NACIONES Y LAS NACIONES UNIDAS}

La SDN, precursora de las NNUU, fue la primera organización internacional de carácter universal. Fue creada mediante el Tratado de Versalles el 28 de junio de 1919, y se basa en el concepto de seguridad colectiva. Tanto la idea de su creación como la posibilidad de llevarla a la práctica fue obra del Presidente Wilson, quien en su discurso al Senado de los EEUU el 22 de enero de 1917, explicó la necesidad de crear una "Liga para la paz". En el último de sus catorce puntos del discurso al Congreso el 8 de enero de 1918 incluye la formación de una "Asociación General de Naciones". Inspirándose en la teoría del Presidente Wilson, el Tratado de Versalles recoge en su articulado el Pacto de La Sociedad de Naciones, que entra en vigor el mismo día que el Tratado, el 10 de enero de 1920. Este nuevo organismo no fue capaz de cumplir satisfactoriamente con la alta misión que se le había encomendado ${ }^{3}$. La II Guerra Mundial estalló, y ni Polonia, ni Francia ni el resto de las naciones decidieron implicar a la SDN en la guerra. La SDN era ya una Institución inoperante cuando en 1940, ante la amenaza de la invasión alemana o italiana, los diplomáticos de la misma decidieron abandonar los cuarteles generales de la organización en Ginebra (Janis, 2003:203-207). Finalmente en 1945 la asamblea de la SDN decidió por unanimidad disolver la organización y transferir todos sus poderes a las NNUU. De igual manera que tras la derrota de Napoleón, la Cuádruple Alianza trató de determinar cómo se podría crear una institución que salvaguardara la paz tras la guerra, en 1945 se buscó la creación de un sistema internacional de cooperación garante de la paz (Buzan y Waever, 2003:123).

El colapso de la SDN y los horrores de la II Guerra Mundial persuadieron a muchos gobiernos sobre la necesidad de crear un sistema internacional que garantizara la seguridad en el periodo posbélico. En 1944 los Estados Unidos, la Unión Soviética, Gran Bretaña y China establecieron las bases y trabajaron conjuntamente para que en 1945 se reunieran cincuenta Jefes de Estado, acordaran la creación de la las NNUU y redactaran la Carta de las Naciones Unidas, que sería firmada por los cincuenta Estados el 24 de octubre de 1945 en San Francisco, California, Estados Unidos. 
La nueva organización multilateral tenía la pretensión de mejorar la SDN en diferentes aspectos, entre los que destaca el que se le otorgara a la nueva institución un poder mayor del que tenía la SDN. Además fueron incluidos en esta organización un mayor número de países que en la SDN, entre ellos Estados Unidos. La nueva institución asumió responsabilidades en muchísimos campos de actuación y otorgó a sus dirigentes un mayor poder e independencia. El objetivo principal de esta nueva institución fue el de evitar a toda costa una tercera guerra mundial (Janis, 2003:208).

La declaración de objetivos de las NNUU puede leerse en el preámbulo de la Carta de las Naciones Unidas. La disposición más importante en términos de seguridad es el Artículo 2.4. ${ }^{4}$. Las guerras posteriores a 1945 han demostrado que, en la práctica, este artículo que prohíbe o restringe el uso de la fuerza, no ha resultado ser más efectivo que el fútil pacto de Briand-Kellogg 5 . La lentitud de actuación del CSNU ha permitido el comienzo de numerosos conflictos desde 1945, amparándose la respuesta militar en el artículo 51 de la Carta de las NNUU6 (Wright, 1963 y Janis, 2003:213).

Las NNUU son mucho más eficientes en evitar el desarrollo de conflictos a nivel global que la SDN, debido a que su estructura institucional facilita enormemente la resolución diplomática de los conflictos. Sin embargo, durante la Guerra Fría se demostró que las NNUU tampoco es una organización eficaz para garantizar la paz mundial, debido al poder de veto de cinco de las naciones integrantes. Por consiguiente, puede decirse que si bien las NNUU ofrecen un lugar de encuentro diplomático y un foro multicultural adecuado para el intercambio de ideas y búsqueda de soluciones técnicas, falla en cumplir con su misión primordial de garantizar la seguridad colectiva, la prevención de la guerra y la oposición internacional automática (Kissinger, 1994:249-250).

Hay que observar el cambio producido en el concepto de "soberanía nacional" y el papel de las naciones en las organizaciones internacionales. Durante los últimos siglos el orden internacional estaba basado en el concepto de la soberanía nacional, en el que cada Estado era el único responsable de custodiar su territorio y de su seguridad nacional. Cuando la SDN se fundó, los firmantes decidieron establecer, en el seno de la organización, una igualdad de soberanía entre los Estados participantes, otorgando a cada uno de ellos una capacidad de decisión alícuota. Fue precisamente este aspecto el que hizo que Estados Unidos no se adhiriera al organismo internacional que había propuesto crear. Sin embargo cuando se crearon las Naciones Unidas en 1945, los Estados decidieron que el desigual poder de los Estados debería estar reflejado de alguna manera en la organización para poder desempeñar eficientemente su misión, aunque sin perjudicar el principio de igualdad de soberanía de cada Estado.

Como resultado de este doble y quizás contradictorio objetivo, llegaron al acuerdo de dotar de una capacidad de voto equitativa a todos los Estados dentro de la Asamblea General de las Naciones Unidas, y el derecho a veto, dentro del Consejo de Seguridad de las Naciones Unidas, para las cinco potencias vencedoras 
en la II Guerra Mundial: Estados Unidos, Francia, el Reino Unido, Rusia y China. Según algunos autores esta fórmula puede interpretarse como el reconocimiento tácito de que la seguridad global no existe y otorga a un pequeño grupo de países la capacidad de condenar a otros a la guerra o garantizarles la paz (Brzezinski, 2004:7-8).

\section{SITUACIÓN INTERNACIONAL TRAS LA II GUERRA MUNDIAL}

El escenario internacional impuesto después de la II Guerra Mundial por los vencedores se basaba en la búsqueda del establecimiento de unas bases sólidas para unas relaciones internacionales fluidas tanto desde el punto de vista político como económico, con el objetivo final de evitar a toda costa una tercera guerra mundial.

Tanto Estados Unidos como Gran Bretaña realizaron grandes esfuerzos diplomáticos para diseñar un nuevo orden mundial caracterizado por una seguridad colectiva eficiente, y unas relaciones económicas entre los países lo más abiertas posibles. El avance más significativo del nuevo orden mundial que se pretendía establecer respecto al que se diseñó en el Tratado de Versalles, fue el de restablecer y potenciar el intercambio comercial entre los Estados, para aprovechar los beneficios derivados del comercio internacional y una economía mundial multilateral que había estado bloqueada desde principios del siglo veinte.

En consecuencia, Alemania y otros Estados vencidos durante la II Guerra Mundial estaban invitados a participar en la nueva economía y en el nuevo orden mundial. Para potenciar este nuevo modelo económico mundial, y el desarrollo económico de todos los Estados, en julio de 1944 durante la Conferencia de Bretton Woods fueron creadas, entre otras, dos de las instituciones económicas internacionales más importantes: El Fondo Monetario Internacional y el Banco Mundial, que tendrían desde entonces un papel tan relevante para el comercio internacional como el del Acuerdo General sobre Comercio y Aranceles creado por la Carta de la Habana en 1947, organismo precursor de la Organización Mundial del Comercio (Gilpin, 1995:5-6).

Desde entonces el concepto de defensa colectiva que representa la acción conjunta contra las agresiones a un Estado entró a formar parte del sistema de seguridad global, e implicaba tanto las acciones militares como otro tipo de acciones (Neuhold, 1997:42). Las alianzas entre naciones habían sido hasta el momento la representación más tradicional de la cooperación internacional en materia de seguridad nacional, promoviendo un acuerdo de respuesta conjunta ante el ataque de los intereses de cualquiera de las dos partes por un adversario común. (Maull, 2005: 13). Sin embargo, estas alianzas entre un pequeño grupo de Estados implican costes económicos y militares, además de la incertidumbre sobre la futura intervención del país aliado en caso de necesitar de su ayuda, y la frecuente subordinación a la voluntad de las decisiones del Estado más poderoso. Estas alianzas suelen crearse ante una amenaza común, como sucedió durante el periodo 
de la Guerra Fría. Una vez la amenaza se ha disuelto los Estados deben evaluar los beneficios e inconvenientes derivados de sus acuerdos y obligaciones militares con sus aliados (Neuhold, 1997:50-51).

\section{LA ORGANIZACIÓN DEL TRATADO DEL ATLÁNTICO NORTE}

La principal organización de defensa europea es la Organización del Tratado del Atlántico Norte, cuyo acrónimo es OTAN ${ }^{7}$. Fue fundada el 4 de abril de 1949. La OTAN cohesiona y organiza a los países aliados en materia política, económica y militar. Nace con el objetivo de organizar la defensa de Europa después de la II Guerra Mundial ante la amenaza de la Unión de Repúblicas Socialistas Soviéticas, en adelante URSS, Estado que igualmente constituyó una organización paralela por medio del Pacto de Varsovia.

La OTAN garantiza un espacio de seguridad común para sus Estados miembros y asegura la defensa de todos los Estados firmantes, asegurando la respuesta conjunta ante una agresión o una amenaza de agresión a un Estado miembro, al entender que la agresión ante uno o varios Estados es la agresión a todos en su conjunto. Los tres objetivos principales para su creación fueron el de contener la posible expansión de la URSS en la Europa del Este y el de garantizar un espacio de seguridad común que permitiese el desarrollo económico y los valores democráticos (OTAN, 2006:15-17).

Un año antes de la fundación de la OTAN, Europa había creado dentro del marco del Tratado de Bruselas de 1948, la Organización Europea de Defensa y Seguridad, formada inicialmente por Francia, Bélgica, Reino Unido, Luxemburgo y Países Bajos. Su objetivo era, en primer lugar, el de asistir a cualquiera de sus miembros en caso de sufrir una agresión, y en segundo lugar potenciar la cooperación e integración europea, objetivos compartidos con el Consejo de Europa, y las diferentes Comunidades Europeas, embriones de la actual Unión Europea, en adelante UE.

Al finalizar la II Guerra Mundial los países europeos que habían quedado fuera del bloque comunista crearon una organización común de defensa para protegerse de un posible ataque de los países de la órbita comunista. Por otro lado los países del Bloque del Este firmaron en 1955 un acuerdo de cooperación militar llamado "El Tratado de Amistad, Colaboración y Asistencia Mutua", más comúnmente conocido como el Pacto de Varsovia. Su objetivo expreso fue el de contrarrestar la amenaza que suponía el establecimiento en 1949 de la OTAN, y especialmente el rearme de la República Federal Alemana (Farmer, 2000:278).

Con la disolución oficial tanto del Pacto de Varsovia en Praga el 1 de julio de 1991, como de la propia URSS tras la declaración de disolución de Gorbachov el 25 de diciembre de 1991, había desaparecido el enemigo común de los países aliados a la OTAN. Muchos analistas vaticinaron una disminución de los gastos militares de los Estados miembros, sin embargo el nacimiento de otros conflictos en la antigua Yugoslavia y en algunas regiones de la antigua URSS provocaron el 
incremento en el gasto militar de algunos países de la $\mathrm{OTAN}^{8}$. Por otro lado surgen nuevas tareas asignadas a las Fuerzas Armadas que también implican la necesidad de nuevas partidas presupuestarias, tales como la asistencia a misiones de paz y la reorganización de la estructura militar para adaptarse a los nuevos desafíos globales (OTAN, 2006:15-17).

Desde el final de la Guerra Fría la OTAN se ha reestructurando, reorientando sus actividades en las siguientes cuatro áreas: adaptación interna, adaptación externa, mantenimiento de la paz y gestión de las crisis. La adaptación interna se refiere a su reestructuración y reforma, que se han basado en reequilibrar las relaciones entre los Estados miembros. Uno de los elementos más importantes de la reestructuración interna fue la creación de estructuras y procedimientos operacionales que permitieran a los Estados europeos de la alianza emprender y asumir la ejecución de operaciones militares sin la participación de las fuerzas armadas norteamericanas. Esto fue posible al crearse en enero de 1994 el acuerdo por el cual se permitía el establecimiento de las Fuerzas Operativas Combinadas Conjuntas. Este acuerdo permite que unas fuerzas armadas operativas y flexibles puedan ser desplegadas al margen de las operaciones establecidas en el artículo quinto ${ }^{9}$. Además ofrece también, la posibilidad de crear una coalición voluntaria de fuerzas armadas de los Estados miembros para realizar actuaciones militares conjuntas dentro del marco de la OTAN, aunque estas actuaciones no respondan al ataque directo sufrido por uno de sus Estados miembros. Por lo tanto en las actuaciones de las Fuerzas Operativas Combinadas Conjuntas no participan todos los Estados miembros, aunque se presupone la aprobación y el respaldo de toda la Alianza (Smith y Timmins, 2001:62-64).

El segundo aspecto en el que ha evolucionado la OTAN es en su relación con los Estados no miembros, especialmente desde 1994 con su participación en procesos de paz, y desde 1997 en sus relaciones con Rusia, que se institucionalizaron con la creación del Consejo Conjunto Permanente entre la OTAN y Rusia. Dentro de las actuaciones de la OTAN en relación al mantenimiento de la paz destaca su actuación en la antigua Yugoslavia y en Bosnia en los años noventa.

Cuando la OTAN lanzó en 1999 la operación de fuerzas aliadas contra los serbios suscitó una gran controversia al no esperar o contar con el visto bueno del Consejo de Seguridad de las Naciones Unidas. Estas actuaciones en terceros países que no están amparadas por el artículo cinco confirmaban de facto que la OTAN estaba asumiendo un nuevo papel en el establecimiento del orden y la paz a nivel mundial, otorgándose la capacidad para ejercer el uso de la fuerza en terceros países en búsqueda del mantenimiento de la paz, papel que asumió oficialmente la OTAN en la Cumbre de Washington en abril de 1999. 


\section{EL TRATADO DE AMISTAD FRANCO-ALEMÁN}

Después de la II Guerra Mundial el desarrollo del marco institucional internacional en Europa se estableció en base a acuerdos de seguridad. En estos acuerdos siempre estaba presente en la memoria de los firmantes, tanto los efectos devastadores de las dos Guerras Mundiales como la amenaza soviética y el deseo y necesidad de recuperación económica del viejo continente. La estrategia primordial de las potencias europeas fue la unión de sus fuerzas, debido a que por ejemplo, tanto Gran Bretaña como Francia tenían escasez de recursos para ser competitivos ante las potencias más importantes de la época (Estados Unidos y Rusia), mientras que por otro lado Alemania tenía parte de su territorio ocupado por las fuerzas aliadas (Brzezinksi, 1997:60, Farmer, 2000:270-278).

El proceso de unificación que se comenzó estaba basado en intereses comunes compartidos y la dependencia mutua para garantizar la seguridad, llevando a los Estados a practicar lo que algunos autores han llamado seguridad cooperativa (Maull, 2005: 15). El punto crucial en el proceso de unificación europeo fue la creación de la Entente entre Francia y Alemania, que estableció los primeros cimientos para la fundación de las instituciones europeas y la futura creación de la Unión Europea (Farmer, 2000:278).

La firma del Tratado de Amistad Franco Alemán en el Palacio del Elysée de París en 1963, fue el principio de unas relaciones diplomáticas y de cooperación política honesta y cercana entre las naciones europeas. Éste ha sido el tratado de amistad y cooperación más importante, más duradero, y con más efectos positivos de cuantos se han firmado entre dos países europeos (Embajada de Alemania en EEUU, 2008).

El concepto de la integración europea ha ofrecido un marco de seguridad y cooperación que ha permitido el desarrollo y creación conjunta de políticas económicas y sociales. Un objetivo primordial de este proceso desde los años setenta ha sido la reducción de la disparidad de poder adquisitivo entre los Estados miembros, con la subsiguiente cohesión económica y social entre las naciones europeas.

Para el correcto funcionamiento de la UE es imprescindible equiparar la capacidad de poder adquisitivo de todas las naciones europeas, eliminando tanto las desigualdades económicas como las sociales y jurídicas. En definitiva, equilibrar el nivel de vida en todos los Estados miembros. Esta premisa no debería ser ignorada por las autoridades económicas y políticas europeas, debido a que una brecha demasiado grande entre el nivel de bienestar y seguridad social de los diferentes países puede provocar un desequilibrio de tal magnitud que sea fuente de tensión o conflicto entre los ciudadanos de las diferentes naciones de Europa (Smith y Timmins, 2001:79). 


\section{ACTUAL MARCO INSTITUCIONAL PARA LA SEGURIDAD EN EUROPA}

Una vez analizado el largo proceso histórico que ha determinado el marco institucional internacional que garantiza la paz en Europa, condición indispensable para el desarrollo pleno del Estado de bienestar y el crecimiento económico sostenido en un ambiente democrático y próspero, se va a analizar su entramado institucional actual.

El siguiente gráfico muestra la visión general del marco institucional internacional para la seguridad del que forman parte los Estados Europeos en la actualidad (Gráfico 1). Una vez concretado el marco institucional internacional para la cohesión y la seguridad en el que participan los Estados europeos se expondrá a continuación de forma concisa la filosofía y el papel que desempeña cada organización internacional.

Gráfico 1. Marco institucional internacional para la cohesión y la seguridad en Europa.

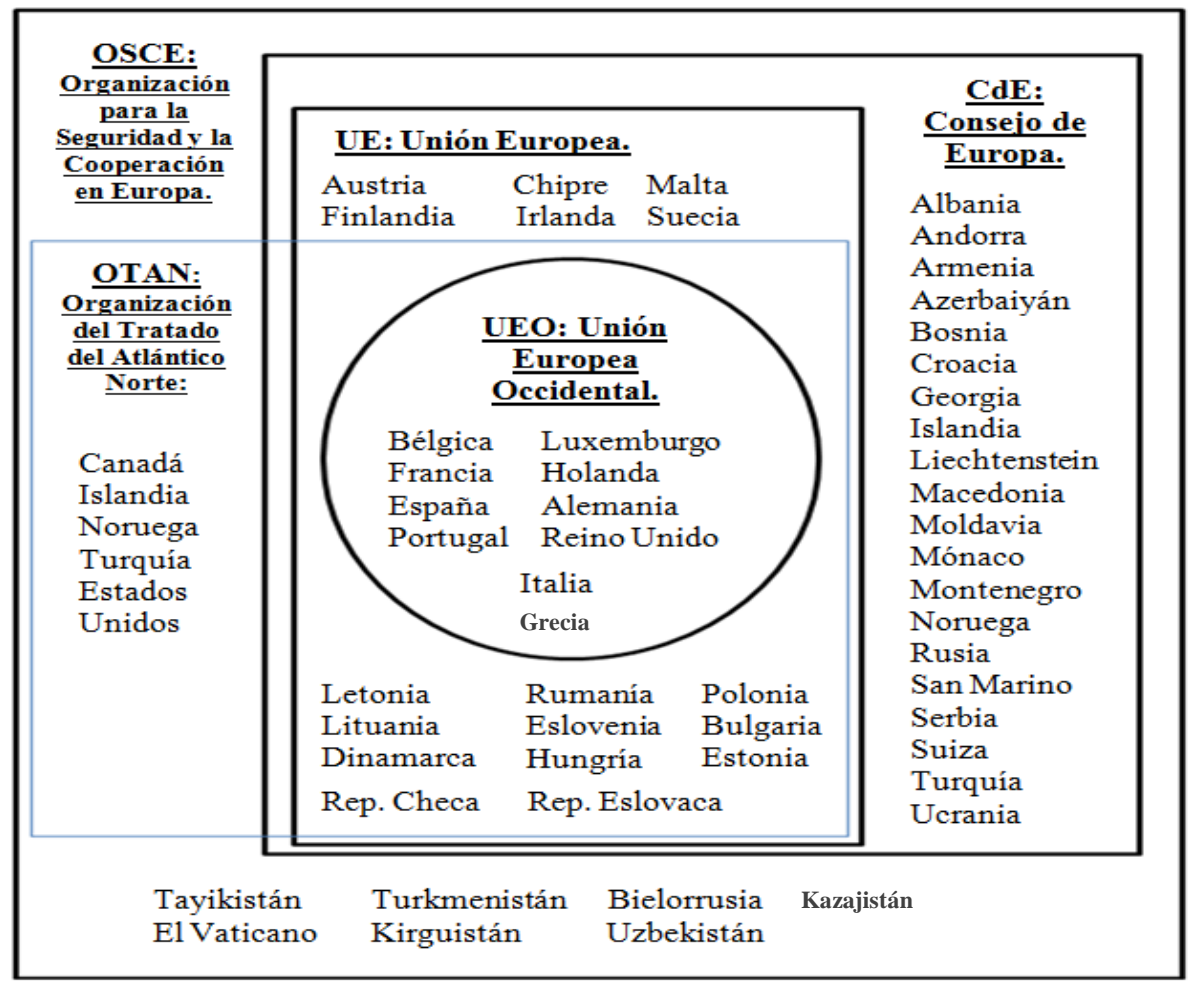

Fuente: Elaboración propia. (F. Blanco y A. Romero, 2008). 


\section{EL CONSEJO DE EUROPA}

El Consejo de Europa es la decana de las instituciones políticas paneuropeas. Desde su fundación por diez Estados en 1949 tiene su sede en Estrasburgo. El objetivo principal de esta organización, tal y como consta en el artículo primero de sus estatutos, es el de lograr una gran unidad entre sus miembros, siendo su misión promover la democracia, proteger los derechos humanos y el Estado de derecho. Busca el desarrollo en común de los principios democráticos basados en la Convención Europea de Derechos Humanos. Además tiene como objetivo el fomento del desarrollo de la diversidad e identidad cultural de los diferentes pueblos de Europa, con especial atención en la promoción de la protección de los derechos individuales de los ciudadanos europeos, respaldando numerosas reformas a nivel político y legislativo.

Las actuaciones más destacadas del Consejo de Europa han sido las medidas políticas adoptadas para erradicar la discriminación contra las minorías, la xenofobia, la intolerancia, el terrorismo, el tráfico de seres humanos, las organizaciones criminales, la corrupción y la violencia doméstica, mediante la creación de cuerpos y comités expertos en materias sociales, legales y culturales. En la actualidad está compuesto por 47 Estados, con una Secretaría General, Comité de Ministros, una Asamblea Parlamentaria, Congresos Locales y Autoridades Regionales (Consejo de Europa, 2008).

\section{LA CONFERENCIA PARA LA SEGURIDAD Y LA COOPERACIÓN EN EUROPA}

La Conferencia para la Seguridad y la Cooperación en Europa, en adelante CSCE, transformada en 1994 en la Organización para la Cooperación y la Seguridad en Europa, en adelante OSCE, tiene su génesis en el periodo de distensión de principios de los años setenta. Su misión constituyente y procedimiento operacional se basaba en la creación de foros multilaterales para el diálogo y la negociación entre la Europa Oriental y la Europa Occidental.

Tras dos años de negociaciones en Ginebra y Helsinki se alcanzó un acuerdo general en 1975, que se plasmó en el Acta Final de Helsinki, firmada el 1 de agosto de 1975. Este acuerdo incluía obligaciones políticas, militares, económicas, medioambientales, y obligaciones vinculadas al respeto de los derechos humanos, que representaron la esencia del Proceso de Helsinki y del Decálogo de Helsinki ${ }^{10}$, que establecía los principios fundamentales que debe respetar todo Estado miembro respecto a la relación con sus ciudadanos y con el resto de los Estados miembros.

La CSCE desempeñaba sus actividades en base a conferencias internacionales hasta el final de la Guerra Fría, cuando en la Cumbre de París de 1990 los Estados miembros firmaron la Carta de París para una Nueva Europa, que consistía en redefinir los objetivos fundacionales, poniendo un mayor énfasis en 
dar respuesta a los desafíos relativos al periodo de transición ulterior a la Guerra Fría, junto con la creación de instituciones permanentes y una nueva capacidad operativa. En la Cumbre de Jefes de Estado de Budapest en 1994 se tomó la decisión de transformar la CSCE en la OSCE.

\section{LA ORGANIZACIÓN PARA LA COOPERACIÓN Y LA SEGURIDAD EN EUROPA}

La OSCE es la mayor organización para la seguridad del mundo, agrupa a 56 Estados y cuenta con un presupuesto que en el año 2008 ascendía a 164 millones de euros (OSCE, 2008).

La organización basa sus actividades en el principio de seguridad cooperativo, cuyo prerrequisito es la necesidad de consentimiento de todas las partes implicadas, incluida la parte en la que se está desarrollando el conflicto. Las medidas de cooperación están basadas en el interés común por el mantenimiento de la seguridad y la búsqueda de soluciones pacíficas (Smith y Timmins, 2001:44).

Respecto al papel de la OSCE en materia de seguridad e intervención en conflictos hay que destacar su gran flexibilidad y su capacidad para actuar en diferentes niveles dentro del concepto de seguridad cooperativa, tales como la prevención y resolución de conflictos, operaciones de mantenimiento de paz, reconstrucción postbélica, y control de armamentos y desarme (Neuhold, 1997:4143).

La OSCE suministra el marco de seguridad y cooperación entre los Estados europeos y las normas fundamentales para el comportamiento tanto en política interior como en política exterior de dichos estados, que tienen un fuerte compromiso político en seguir las directrices impuestas por la OSCE. La ausencia de represalia por parte de la OSCE ante los países infractores y su incapacidad para establecer un régimen de seguridad cuando se transgrede ilustra la mayor debilidad de esta organización. A pesar de sus limitaciones es el mejor exponente del régimen de seguridad global del que forman parte los Estados europeos (Smith y Timmins, 2001:51-54).

Desde su refundación en Budapest en 1994, y principalmente tras las Cumbres de Lisboa 1996 y Estambul 1999, la OSCE ha trabajado arduamente por incrementar los compromisos adquiridos y adherir a su régimen a los países de la extinta URSS. Los sucesos acontecidos en la antigua Yugoslavia y en algunos de los Estados creados a partir de la balcanización de la URSS han demostrado que la OSCE no ha sido capaz de cumplir satisfactoriamente con su alta misión de garante de la paz y la seguridad.

Por lo tanto son la UE y la OTAN, en mayor medida que la OSCE, las principales instituciones multilaterales que garantizan tanto la paz como la cohesión de los Estados europeos. Hay que destacar que a pesar de que las instituciones europeas han conseguido alcanzar un privilegiado nivel de paz y seguridad en prácticamente la totalidad del continente, incluida la Europa del este, 
existen dos áreas donde todavía no se ha conseguido este escenario idílico, representando los Balcanes y algunos países de la antigua URSS la principal asignatura pendiente para Europa.

\section{LA UNIÓN EUROPEA}

La contribución principal de la UE a la paz y seguridad en Europa es la creación de un entramado institucional y una cohesión política y económica de tal magnitud que ha conseguido el establecimiento de fuertes vínculos económicos, políticos y culturales entre los pueblos europeos. Gracias a las políticas adoptadas por las diferentes instituciones de la UE se ha potenciado el crecimiento económico de todos los Estados ${ }^{11}$. Desde los años cincuenta hasta el final de la Guerra Fría la integración en Europa se basó únicamente en términos económicos. Las Comunidades Europeas ${ }^{12}$, precedentes de la actual UE, fueron tres organizaciones internacionales de cooperación económica.

La CECA se constituyó en 1951 y fue la semilla de la futura UE, organización a la que traspasó sus actividades al extinguirse su periodo de validez en 2002. Tanto la CEE como la CEEA fueron creadas por la firma de los Tratados Fundacionales de Roma de 1957.

La más importante de las Comunidades Europeas fue la CEE, que durante sus primeros años de existencia obtuvo logros importantes en materia de política económica, entre los que destaca el establecimiento de un arancel exterior conjunto, una política agrícola común y la creación de instituciones para el desarrollo económico.

En el año 1992, en Maastricht, se firma el Tratado de la Unión Europea que entra en vigor el 1 de noviembre de 1993, nacimiento de la nueva Unión Europea, institución de la que pasan a forman parte las tres Comunidades Europeas. Asimismo la CEE pasó a llamarse Comunidad Europea, que constituye el eje principal de la nueva UE.

Las constantes ampliaciones de la UE, que en la actualidad cuenta con 27 Estados miembros, junto con la gran variedad e importancia de los acuerdos alcanzados en política económica, que incluyen aspectos tan transcendentes como la libre circulación de mercancías, personas, servicios y capitales desde 1986, o la creación de una Unión Económica y Monetaria con la implantación de una moneda única en el 2002, representan la contribución esencial de la UE a la paz como consecuencia directa de la estabilidad política y la prosperidad económica en Europa.

\section{LAS POLÍTICAS EN SEGURIDAD Y DEFENSA DE LA UE}

La política europea de seguridad y defensa tiene sus orígenes en los años cincuenta. Además de incentivar la integración económica entre Francia y Gran Bretaña para evitar una nueva guerra, se estableció, en 1952, la Comunidad 
Europea de la Defensa, en adelante CED, con el objetivo de integrar a Alemania del Este dentro del sistema de defensa europeo y fortalecer el este de Europa ante un hipotético ataque soviético. Sin embargo la CED fracasó y se disolvió dos años después de su creación, por lo que la cooperación en materia de seguridad volvía a depender en exclusiva de la OTAN y de la Unión Europea Occidental (Peterson, 2006:254-255). La UEO es desde su creación por el Tratado de Bruselas de 1948, la organización de defensa y seguridad del ámbito europeo formada por los estados miembros de la Unión Europea y los miembros europeos de la OTAN.

En 1970 se crea el mecanismo de cooperación político europeo encargado de la política exterior de la UE. Este organismo alcanzó finalmente un estatus institucional tras la primera gran reforma de los Tratados de París y Roma en los Tratados del Acta Única Europea en Luxemburgo y La Haya en 1986. Incluso tras cambiar su estatus siguió siendo ineficaz como institución gestora de los problemas europeos en materia de política exterior, tal y como se demostró durante los conflictos étnicos en Yugoslavia y en la primera guerra de Iraq. En el Tratado de Maastricht de 1992 se incluyó dentro de la estructura institucional de la UE, pasando a constituir la base del segundo de los tres pilares de la UE, y siendo conocido desde entonces como Política Exterior de Seguridad y Defensa Común, en adelante PESC.

A pesar de la creación, por el Tratado de Ámsterdam de 1997, del puesto de Alto Representante para la PESC, que tiene la misión de contribuir a la formulación de la política exterior comunitaria y es el encargado de asistir al Consejo en las cuestiones que correspondan a la PESC, no se consiguió mejorar el funcionamiento de la misma, dada la ineficiencia institucional y militar europea para gestionar una situación de crisis sin el apoyo de Estados Unidos, tal y como quedó humillantemente demostrado durante el último conflicto en los Balcanes (Peterson, 2006:255-259).

La política exterior y de seguridad común de la UE busca una progresiva política común de defensa que podría conducir, llegado el momento, a una defensa común efectiva. La PESC tiene por objetivo permitir a la UE desarrollar sus capacidades civiles y militares de gestión de las crisis y de prevención de los conflictos a escala internacional, aunque no implica la creación de un ejército europeo. La PESC es compatible y se coordina con la OTAN (Unión Europea, 2008).

La defensa autónoma y operativa de la UE en la actualidad la forman el Comité político y de seguridad, COPS, el Comité militar de la UE, CMUE, y el Estado Mayor militar de la UE, EMUE. El Consejo Europeo de Helsinki, celebrado en diciembre de 1999, instauró el llamado objetivo global, que consiste en la capacidad de la UE para poder desplegar hasta 60.000 hombres en el plazo máximo de 60 días y durante al menos un año. En el Consejo Europeo de Gotemburgo en 2001 se expresó la voluntad de mejorar las capacidades de la UE en los ámbitos de la prevención de los conflictos y de la gestión de las crisis con medios militares y civiles. Por el Tratado de Niza de 2001, se atribuyó al COPS la tarea de las 
operaciones de gestión de las crisis, bajo la responsabilidad del Consejo (Unión Europea, 2008).

\section{CONCLUSIÓN}

A lo largo del presente artículo se ha expuesto la historia institucional y geopolítica europea en materia de seguridad. Se han analizado los conceptos y estudiado las causas de la evolución hacia el marco institucional actual. Como se ha observado son muchos y muy profundos los cambios que se han producido en el viejo continente desde la Paz de Westfalia. Desde el siglo XVII cuando las relaciones internacionales europeas estaban basadas en el principio de soberanía nacional hasta el siglo XXI, en el que el concepto de defensa está basado en un marco multilateral de seguridad global que vela por la defensa de los derechos humanos y la paz global. De hecho, los tres pilares de la UE sobrepasan el principio de soberanía nacional de Westfalia transfiriendo la gestión de parte de su soberanía a las Instituciones Europeas, con el objetivo de una mayor integración y cohesión entre los 27 Estados miembros de la UE.

El continente europeo alberga en la actualidad el entramado institucional más multilateral y complejo del mundo, que como se ha mostrado a lo largo de este artículo, es el resultado de una larga y difícil evolución histórica, en la que todos los Estados europeos han sufrido durante siglos las consecuencias de la destrucción mutua y sus crisis económicas consiguientes.

Con la aparición de la idea de Estado-Nación, tras la Paz de Westfalia, se instauró el sistema de congresos internacionales entre las grandes potencias para la resolución de los conflictos, creando el concepto de legitimación de acción conjunta, precursor del presente sistema institucional internacional. Con el objetivo común de terminar con la espiral de violencia y destrucción que supusieron las dos Guerras Mundiales, los Estados europeos priorizaron la creación tanto de relaciones económicas entre los diferentes países como de instituciones de seguridad colectivas como la Liga de las Naciones, posterior Naciones Unidas, instituciones de defensa colectivas como la Organización del Tratado del Atlántico Norte e instituciones para la seguridad cooperativa y para la cohesión de los Estados europeos como el Consejo de Europa, la Organización para la Cooperación y la Seguridad en Europa y la Unión Europea.

\section{NOTAS}

${ }^{1}$ Que era el principal objetivo de Carlos I de España y V de Alemania. Esta idea se deshizo con su abdicación en 1555 y la división de su herencia entre su hijo Felipe II (monarquía hispánica) y su hermano Fernando I (Imperio alemán). 
${ }^{2}$ La Alianza de la Haya consiguió en el Tratado de Rastadt que Felipe V renunciara a la sucesión del trono francés heredado de su abuelo Luis XIV. Si se hubieran unido Francia y España sí hubieran sido la potencia dominadora de Europa.

${ }^{3}$ Siendo incapaz, por ejemplo, de impedir la invasión de Manchuria por Japón en 1931, la invasión de Etiopía por Italia en 1934, o promoviendo demasiado tarde la intervención francesa e inglesa cuando Rusia y Alemania atacaron Polonia en 1939.

${ }^{4}$ El Artículo 2.4 de la Carta de las NNUU establece que "los miembros de la Organización, en sus relaciones internacionales, se abstendrán de recurrir a la amenaza o al uso de la fuerza contra la integridad territorial o la independencia política de cualquier Estado, o cualquier otra forma incompatible con los Propósitos de las Naciones Unidas".

${ }^{5}$ En el pacto de Briand-Kellogg, conocido también como Pacto de París firmado el 27 de agosto de 1928, quince signatarios renunciaron a la guerra "como instrumento de política nacional en sus relaciones mutuas".

${ }^{6}$ El artículo 51 de la Carta de las NNUU establece que "Ninguna disposición de esta Carta menoscabará el derecho inmanente de legítima defensa, individual o colectiva, en caso de ataque armado contra un Miembro de las Naciones Unidas, hasta tanto que el Consejo de Seguridad haya tomado las medidas necesarias para mantener la paz y la seguridad internacionales".

${ }^{7}$ La OTAN fue creada como resultado de las negociaciones entre los signatarios del Tratado de Bruselas, es decir, Bélgica, Francia, Luxemburgo, Holanda y el Reino Unido, además de Estados Unidos y Canadá, así como otros cinco países de Europa Occidental invitados a participar: Dinamarca, Italia, Islandia, Noruega y Portugal. España se adhirió a la OTAN el 30 de mayo de 1982.

${ }^{8}$ Para un mejor entendimiento de los determinantes del gasto en defensa de los Estados miembros de la OTAN debe consultarse el siguiente texto de referencia dentro de la literatura especializada: "Los determinantes del gasto militar en los países europeos de la Alianza Atlántica: un estudio a través de un modelo de datos de panel para el período 1975-2005", cuyos autores son el Prof. Alonso Neira y el Prof. Martínez González (IX Reunión de Economía Mundial, 2007).

${ }^{9}$ Artículo 5 de la OTAN: "Las Partes acuerdan que un ataque armado contra una o más de ellas, que tenga lugar en Europa o en América del Norte, pueda ser considerado como un ataque dirigido contra todas ellas, y en consecuencia, acuerdan que si tal ataque se produce, cada una de ellas, en ejercicio del derecho de legítima defensa individual o colectiva reconocido por el artículo 51 de la Carta de las Naciones Unidas, pueda ayudar a la Parte o Partes atacadas, adoptando seguidamente, de forma individual y de acuerdo con las otras Partes, las medidas que juzguen necesarias, incluso el empleo de la fuerza armada, para restablecer la seguridad en la zona del Atlántico Norte. Cualquier ataque armado de esta naturaleza y todas las medidas adoptadas en consecuencia serán inmediatamente puestas en conocimiento del Consejo de Seguridad. Estas medidas cesarán cuando el Consejo de Seguridad haya tomado las disposiciones necesarias para restablecer y mantener la paz y la seguridad internacionales."

${ }^{10}$ El Decálogo de Helsinki consta de los siguientes puntos: Igualdad soberana y respeto de los derechos inherentes a la soberanía; Abstención de recurrir a la amenaza o al uso de la fuerza; Inviolabilidad de las fronteras; Integridad territorial de los Estados; Arreglo de las controversias por medios pacíficos; No intervención en los asuntos internos; Respeto de los 
derechos humanos y de las libertades fundamentales, incluida la libertad de pensamiento, conciencia, religión o creencia; Igualdad de derechos y libre determinación de los pueblos; Cooperación entre los Estados; Cumplimiento de buena fe de las obligaciones contraídas según el derecho internacional (CSCE, 1975).

${ }^{11}$ Esta idea queda perfectamente definida en la célebre frase del economista francés Frederic Bastiat: "Si las mercancías no cruzan las fronteras, lo harán los soldados". A esta relación inversa se la conoce como "efecto Bastiat" (Alonso y Martínez, 2007).

${ }^{12}$ Las tres Comunidades Europeas son la CECA, Comunidad Europea del Carbón y del Acero, la CEE, Comunidad Económica Europea, y la CEEA o EURATOM, Comunidad Europea de la Energía Atómica.

\section{BIBLIOGRAFÍA}

ALONSO, M. A. y MARTÍNEZ, A. (2007): "Los determinantes del gasto militar en los países europeos de la Alianza Atlántica: un estudio a través de un modelo de datos de panel para el período 1975-2005", Economía Mundial, 9:209-246. Disponible en Internet: www.uam.es/otros/ixrem/Comunicaciones/Area\%2 012-Economicas\%20nacionales.pdf [Consulta: 28 abril 2008].

BAILES, A. J. K. (2005): “The European Security Strategy”, en SIPRI Policy Paper, 10: 2-46. Disponible en Internet: www.sipri.org/contents/editors/publications/ESS_PPrapport.pdf [Consulta: 20 abril 2008]

BEN-ARI, G., MCINNIS, K. y SCRUGGS, D. (2005): European Defence Integration: Bridging the Gap between Strategy and Capabilities, Washington, D.C., CSIS ; Disponible en Internet: www.csis.org/media/csis/pubs/0510_eurodefensereport.pdf [Consulta: 20 abril 2008] BRZEZINSKI, Z. (2004): The Choice: Global Domination or Global Leadership, New York, Basic Books.

- (1997): The Grand Chessboard, New York, Basic Books.

BUZAN, B. y WAEVER, O. (2003): Regions and Powers: The Structure of International Security, Cambridge, Cambridge University Press.

COHRS, P. O. (2006): "The unfinished Peace after World War I: America, Britain and the Stabilization of Europe, 1919 - 1932", Cambridge University Press, 1: 1-22. Disponible en Internet: assets.cambridge.org/97805218/53538/excerpt/9780521853538_excerpt.pdf [Consulta: 24 abril 2008]

CONSEJO DE EUROPA. (1999): Texto refundido del convenio para la protección de los derechos y de las libertades fundamentales, hecho en Roma el 4 de noviembre de 1950. Disponible en Internet: constitucion.rediris.es/legis/1950/tr1950-11-04_roma.html [Consulta: 27 abril 2008]

CSCE. (1990): Carta de París para una Nueva Europa. Disponible en Internet: www.osce.org/documents/mcs/1990/11/4045_es.pdf [Consulta: 24 abril 2008]

DÍEZ ESPINOSA, J. R. (1994): Sociedad y cultura en la República de Weimar: el fracaso de una ilusión Valladolid, Universidad de Valladolid.

DOSCH, J. (2003): Changing Security cultures in Europe and Southeast Asia: Implications for inter-regionalism, en Asia Europe Journal, 1: 1-19. Disponible en Internet: www.smlc.leeds.ac.uk/eas/ 
eas_content/about/floats/staff_details/documents/10308051.pdf.

EMBAJADA DE ALEMANIA en EEUU. (2008): “The Treaty - Foundation for a Special Relationship", Disponible en Internet: www.germany.info/relaunch/info/publications/ infocus/40Elysee/overview.html [Consulta: 20 abril 2008]

EVERS, F. (2002): Building Co-operation between OSCE Field Missions and Partner Institutions in the Economic and Environmental Dimension, Hamburg, Institute for Peace Research and Security Policy.

FARMER, A. (2000): An Introduction to Modern European History: 1890-1990, London, Hodder \& Stoughton.

FERRO, M. (1998): La Gran Guerra (1914-1918), Madrid, Alianza.

GILPIN, R. (1995): "APEC in a New International Order", en NBR Analysis, 6: 5-24. Disponible en Internet: www.nbr.org/publications/analysis/pdf/vol6no5.pdf [Consulta: 20 abril 2008]

GNESOTTO, N. (2005): A Secure Europe in a Better World, Brussels, European security strategy. Disponible en Internet: ue.eu.int/uedocs/cmsUpload/78367.pdf [Consulta: 26 abril 2008]

HOBSBAWM, E. J. (1995): Historia del siglo XX (1914-1991), Barcelona, Crítica.

JANIS, M. W. (2003): An Introduction to International Law, New York, ASPEN Publishers.

JOSHUA, H. y MANJEET, S. (2006): Globalization and Defense, Singapore, Institute of Defense and Strategic Studies.

KEYNES, J. M. (1919): Las consecuencias económicas de la paz, Barcelona, Crítica.

KISSINGER, H. (1994): Diplomacy, New York, Rockefeller Centre.

MARIÑO, F. (1975): "Seguridad y cooperación en Europa: el acta final de Helsinki", en Derecho Internacional, UAM 3: 639-659. Disponible en Internet: www.cepc.es/rap/Publicaciones/Revistas/5/ RIE_002_003_007.pdf [Consulta: 28 abril 2008] MAULL, H. W. (2005): "Security Cooperation in Europe and Pacific Asia: A Comparative Analysis", The Journal of East Asian Affairs, 19: 67-108. Disponible en Internet: www.politik.uni-trier.de/mitarbeiter/maull/pubs/securitycoop.pdf [Consulta : 26 abril 2008] NEUHOLD, H. (1997): "Kooperative Sicherheit - Kollektive Sicherheit - Kollektive Verteidigung - Eine Bestandsaufnahme aus europäischer Sicht". Disponible en Internet www.bmlv.gv.at/pdf pool/publikationen/03 jb97_03.pdf. [Consulta: 20 abril 2008]

- (2000): “Collective Security after Operation 'Allied Force'“, Max Plank Max Yearbook of United Nations Law, 4: 73-106. Disponible en Internet www.mpil.de/shared/data/pdf/ pdfmpunyb/neuhold_4.pdf [Consulta: 20 abril 2008]

NNUU. (1945): Carta de las Naciones Unidas, San Francisco, Naciones Unidas. Disponible en Internet www.cinu.org.mx/onu/documentos/cartatxt.htm [Consulta: 19 abril 2008]

OSCE. (2005): OSCE Handbook 2005, Vienna, Secretariat of the Organization for Security and Cooperation in Europe. Disponible en Internet: www.osce.org/publications/osce/ 2005/04/13858_222_en.pdf [Consulta: 28 abril 2008]

- (2008): $706^{\bar{a}}$ Sesión plenaria del Consejo, Decisión № 844. Disponible en Internet: www.osce.org/documents/pc/2008/03/30257_es.pdf [Consulta: 28 abril 2008]

OTAN. (2004): NATO in the 21st Century, Brussels, NATO Public Diplomacy Division. Disponible en Internet:www.nato.int/docu/21-cent/21st_eng.pdf [Consulta: 27 abril 2008] - (2004): NATO Transformation, Bruselas, NATO Public Diplomacy Division. Disponible en Internet: www.nato.int/docu/nato-trans/nato-trans-eng.pdf [Consulta: 19 abril 2008] 
- (2004): NATO's Military Structure, Brussels, NATO Public Diplomacy Division. Disponible en Internet: www.nato.int/docu/briefing/nms/nms-e.pdf [Consulta: 18 abril 2008]

- (2004): Security through Partnership, Brussels, NATO Public Diplomacy Division. Disponible en Internet:www.nato.int/docu/sec-partnership/sec-partner-e.pdf [Consulta: 16 abril 2008]

- (2006): Handbook 2006, Brussels, NATO Public Diplomacy Division. Disponible en Internet: www.nato.int/docu/handbook/2006/hb-en-2006.pdf [Consulta: 26 abril 2008]

PETERSON, J. (2006): The Institutions of the European Union, New York: Oxford University Press.

SMITH, M. A. y TIMMINS G. (2001): Uncertain Europe: Building a New European Security Order?, London, Routledge.

ZELLNER, W. (2005): Managing Change in Europe, Evaluating the OSCE and its Future Role: Competences, Capabilities and Missions, Hamburg, Institute for Peace Research and Security Policy.

\section{Breve currículo:}

\section{Francisco José Blanco Jiménez}

Doctor en Ciencias Económicas y Empresariales por la Universidad Complutense de Madrid con la calificación de Sobresaliente "Cum Laude". Actualmente es Vicerrector de Información y Comunicación de la Universidad Rey Juan Carlos, donde imparte docencia como Profesor Titular de Universidad en el Departamento de Economía Aplicada I. Es miembro de diferentes grupos de investigación y centros de estudios, entre los que destacan el Centro de Estudios de Economía de Madrid y el Centro de Estudios para Iberoamérica. Ha sido miembro del Tribunal de Oposiciones de la Carrera Diplomática y autor de numerosos libros y artículos de investigación, entre los que destacan La integración económica y el medio ambiente y Técnicas de comercio exterior.

\footnotetext{
Alberto Romero Ania

Máster en Análisis Económico Internacional y Licenciado con "Premio Extraordinario" fin de carrera en Administración y Dirección de Empresas por la Universidad Rey Juan Carlos. Actualmente es Profesor en el Departamento de Economía Aplicada I de la Universidad Rey Juan Carlos. Al mismo tiempo estudia el segundo año de un Máster en Dirección de Empresas. Durante el presente curso académico ha realizado dos estancias breves de investigación, la primera en la Unión Internacional de Telecomunicaciones en Suiza, y la segunda en la Universidad de Ciencias Aplicadas de Krems, en Austria. Entre las becas y premios predoctorales destacan la Beca de la Fundación Caja Madrid, para estudios en el extranjero y el Premio al Mejor Estudiante Erasmus de la Universidad Rey Juan Carlos.
} 